\title{
Isomorphic Digraphs from Affine Maps of Finite Cyclic Groups
}

\author{
Guixin Deng \\ School of Mathematical Science, Guangxi Teachers Education University, Nanning 530001, China \\ Correspondence should be addressed to Guixin Deng; dengguixin@live.com
}

Received 27 June 2013; Accepted 28 July 2013

Academic Editors: L. Feng and J. Rada

Copyright (C) 2013 Guixin Deng. This is an open access article distributed under the Creative Commons Attribution License, which permits unrestricted use, distribution, and reproduction in any medium, provided the original work is properly cited.

Let $n$ be a positive integer. For any pair of integers $k$ and $a$, let $G(n, k, a)$ be the digraph whose set of vertices is $\{0,1,2, \ldots, n-1\}$, and there exists a directed edge from vertex $x$ to vertex $y$ if $k x+a \equiv y(\bmod n)$. In this paper, we obtain a necessary and sufficient condition for which $G\left(n, k_{1}, a_{1}\right) \simeq G\left(n, k_{2}, a_{2}\right)$.

\section{Introduction}

Let $X$ be a nonempty set and let $f$ be a mapping from $X$ into itself. Then, we call $G(X, f)$ the iteration digraph from $f$ of $X$, where the set of vertices of $G(X, f)$ is $X$ and there is a directed edge from a vertex $a \in G(X, f)$ to a vertex $b \in G(X, f)$ if $f(a)=b$. In particular, there are many interesting structures on the digraphs when there is an algebraic structure on $X$.

Many people study the iteration graphs from endomorphisms of finite abelian groups. The authors of [1] studied the digraph from the endomorphism $f(x)=x^{2}$ of $(\mathbb{Z} / p \mathbb{Z})^{*}$, where $p$ is a prime. These results were generalized to the digraph from any endomorphism of $(\mathbb{Z} / p \mathbb{Z})^{*}$ in [2]. Sha and $\mathrm{Hu}$ [3] studied some elementary properties of the digraph from any endomorphism of $\mathbb{F}_{q}^{*}$. They also obtained many elementary properties of the digraph from any endomorphism of finite cyclic groups in [3].

Let $n$ be a positive integer and lrt $k, a$ be two integers. In this paper, we denote by $G(n, k, a)$ the digraph obtained from the affine mapping $x \mapsto k x+a$ of $\mathbb{Z}_{n}$, the ring of integers modulo $n$. In particular, we set $G(n, k)=G(n, k, a)$ if $n \mid a$, which is from an endomorphism $x \mapsto k x$.

We are mainly interested in the problem when $G\left(n, k_{1}\right.$, $\left.a_{1}\right) \simeq G\left(n, k_{2}, a_{2}\right)$. The authors in [2] gave an example $G(10,2) \simeq G(10,8)$. Let $p$ be a prime. Sha [4] showed that $G\left(p, k_{1}\right) \simeq G\left(p, k_{2}\right)$ if and only if $\operatorname{ord}_{p} k_{1}=\operatorname{ord}_{p} k_{2}$. In [5], Deng and Yuan gave a necessary and sufficient condition for $G\left(p-1, k_{1}\right) \simeq G\left(p-1, k_{2}\right)$, where $p$ is a prime. In this paper, we obtain a necessary and sufficient condition for when $G\left(n, k_{1}, a_{1}\right) \simeq G\left(n, k_{2}, a_{2}\right)$.
The paper is organized as follows. In Section 2, we study the $G(n, k, a)$, where $n$ is a power of a prime. The main results are proved in Section 3. We also determined the digraph from projective map on one-dimensional projective space over a finite field in the last section.

\section{Digraph Product and the Local Case}

Let $G$ be a digraph and let $a$ be a vertex in $G$. A component of a digraph is a subdigraph which is a maximal connected subgraph. Each component of a finite iteration graph $G(X, f)$ contains a unique cycle. Let $O_{i}$ denote this $i$-cycle. Let $A_{1}(G)$ denote the number of cycle of length 1 in $G$.

Let $G_{1}, G_{2}, \ldots, G_{r}$ be iteration digraphs. The product $G_{1} \times$ $G_{2} \times \cdots \times G_{r}$ is the digraph whose vertices are the ordered pairs $\left(a_{1}, a_{2}, \ldots, a_{r}\right)$, where $a_{i} \in G_{i}$ and there is a directed edge from $\left(a_{1}, a_{2}, \ldots, a_{r}\right)$ to $\left(b_{1}, b_{2}, \ldots, b_{r}\right)$ if and only if there is a directed edge in $G_{i}$ from $a_{i}$ to $b_{i}$ for each $1 \leq i \leq r$. We use the following notation:

$$
\prod_{i=1}^{r} G_{i}:=G_{1} \times G_{2} \times \cdots \times G_{r} .
$$

Lemma 1 (see [6]). $O_{s} \times O_{t}$ is isomorphic to $\operatorname{gcd}(s, t) O_{\mathrm{lcm}[s, t]}$, which is a union of $\operatorname{gcd}(s, t)$ cycles, each having length $1 \mathrm{~cm}[s, t]$.

Theorem 2. Let $n=\prod_{i=1}^{r} p_{i}^{e_{i}}$ be the prime factorization of $n$ and let $k, a$ be two integers. Then

$$
G(n, k, a) \cong \prod_{i=1}^{r} G\left(p_{i}^{e_{i}}, k, a\right) .
$$


Proof. Let $G=G(n, k, a)$ and $G_{i}=G\left(p_{i}^{e_{i}}, k, a\right)$. Assume that

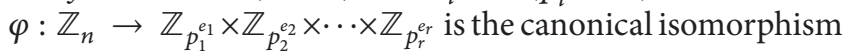
of rings. Then $\varphi$ induces an isomorphism of digraphs.

2.1. The Case of Linear Maps. The structure of $G(n, k)$ has been studied in $[2,4,5]$. We present some results here.

Lemma 3 (see [4]). Let $k, n$ be positive integers. Then one has the following.

(i) If $p \mid k$ for any prime $p \mid n$, then $G(n, k)$ is a tree attached to the fixed point 0.

(ii) If $\operatorname{gcd}(n, k)=1$, then $G(n, k) \simeq \cup_{r} a_{r} O_{r}$, where $a_{r}=$ $(1 / r) \sum_{i \mid r} \mu(i) \operatorname{gcd}\left(n, k^{r / i-1}\right)$ and $\mu$ is the Möbius function.

Let $v_{p}(n)$ be the $p$-adic valuation of $n$ for any prime $p$ and integer $n$. It is the largest power of $p$ dividing $n$ for any prime $p$ and integer $n$. We set $v_{p}(0)=\infty$ as usual. If $X$ is a set of primes and $n$ is a positive integer, we denote that

$$
n_{X}=\prod_{p \in X} p^{v_{p}(n)}
$$

Lemma 4. Let $p$ be a prime. Let $k$, a be positive integers such that $k, a \leq p^{e}-1$. The following statements are equivalent:

(i) $G\left(p^{e}, k, a\right) \simeq G\left(p^{e}, k\right)$;

(ii) $\operatorname{gcd}\left(p^{e}, k-1, a\right)=\operatorname{gcd}\left(p^{e}, k-1\right)$;

(iii) $v_{p}(k-1) \leq v_{p}(a)$;

(iv) $G\left(p^{e}, k, a\right)$ has a fixed point.

Proof. By hypothesis the statements (ii), (iii), and (iv) are equivalent to that the congruence equation $(k-1) x \equiv a(\bmod$ $\left.p^{e}\right)$ that has a solution. Suppose that $G\left(p^{e}, k, a\right) \simeq G\left(p^{e}, k\right)$. Then $G\left(p^{e}, k, a\right)$ has a fixed point $z$ since $G\left(p^{e}, k\right)$ has a fixed point 0 . So $k z+a \equiv z\left(\bmod p^{e}\right)$. On the other hand, if $k z+a \equiv$ $z\left(\bmod p^{e}\right)$, then $x \mapsto x+z$ is an isomorphism of $G\left(p^{e}, k\right)$ and $G\left(p^{e}, k, a\right)$.

Remark 5. Many people showed that the tree attached to any two cycle vertices in $G(n, k)$ is isomorphic. Moreover, Deng and Yuan [5] showed that the tree attached to cycle vertices in $G(p-1, k)$ is totally determined by $\operatorname{gcd}(p-1, k)$, where $p$ is a prime. In fact, if we replace $p-1$ by $n$ in the arguments in [5], then the result is also valid.

\subsection{The Case of Affine Maps}

Lemma 6. Let $k=l p^{t}+1$ be a positive integer, where $p$ is a prime and $p+l, t \geq 1$.

(i) If $p>2$ or $t>1$, then $\operatorname{ord}_{p^{r}} k=\max \left\{1, p^{r-t}\right\}$ for any integers $t \geq 1$.

(ii) If $p=2$ and $t=1$, assuming that $2^{u} \| k^{2}-1$, then

$$
\operatorname{ord}_{2} k= \begin{cases}1, & r=1 \\ 2, & 2 \leq r \leq u \\ 2^{r-u+1}, & r>u\end{cases}
$$

Proof. If $r \leq t$, then it is clear that $\operatorname{ord}_{p^{r}} k=1=\max \left\{1, p^{r-t}\right\}$. Firstly, we assume that $r>t$. By computation, $p^{m-s_{i}} \|\left(\begin{array}{c}p^{m} \\ i\end{array}\right)$ for any $1 \leq i \leq p^{m}-1$ and $p^{s_{i}} \| i$. But

$$
k^{p^{r-t}}-1=\left(l p^{t}+1\right)^{p^{r-t}}-1=\sum_{i=1}^{p^{r-t}}\left(\begin{array}{c}
p^{r-t} \\
i
\end{array}\right)\left(l p^{t}\right)^{i}
$$

thus $p^{r-t-s_{i}+t i} \|\left(\begin{array}{c}p^{r-t} \\ i\end{array}\right)\left(l p^{t}\right)^{i}$. It is clear that $r-t-s_{i}+t_{i} \geq r$. So $p^{r} \mid k^{p^{r-t}}-1$; considering that $\operatorname{ord}_{p^{r}} k \mid p^{r-t}$. So ord $p^{r} k=p$ if $r-t=1$. Suppose that $r-t>1$. If $p>2$ or $t>1$, then

$$
\begin{aligned}
k^{p^{r-t-1}}-1 & =\left(l p^{t}+1\right)^{p^{r-t-1}}-1 \\
& =\sum_{i=1}^{p^{r-t-1}}\left(\begin{array}{c}
p^{r-t-1} \\
i
\end{array}\right)\left(l p^{t}\right)^{i} \\
& =l p^{r-1}+\sum_{i=2}^{p^{r-t-1}}\left(\begin{array}{c}
p^{r-t-1} \\
i
\end{array}\right)\left(l p^{t}\right)^{i} .
\end{aligned}
$$

Therefore, $r-t-1-s_{i}+t_{i} \geq r$ for any $i \geq 2$. Hence, $p^{r}$ | $\left(\begin{array}{c}p^{r-t-1} \\ i\end{array}\right)\left(l p^{t}\right)^{i}$ for $i \geq 2$ and $p^{r}+k^{p^{r-t-1}}-1$. Thus, ord $p^{r} k=p^{r-t}$ in this case.

Suppose that $2 \| k-1$ and $2^{u} \| k^{2}-1, u \geq 3$. It remains to show that $\operatorname{ord}_{2^{r}} k=2^{r-u+1}$ for $r>u$. Let $k^{2}=q 2^{u}+1$, where $2+q$. By the discussion above we get $2^{r} \| k^{2^{r-u+1}}-1$ and $2^{r-1} \| k^{2^{r-u}}-1$. Therefore, $\operatorname{ord}_{2^{r}} k=2^{r-u+1}$ for $r>u$. This finishes the proof.

Lemma 7. Let $p$ be a prime. Let $k, a \leq p^{e}-1$ be two positive integers such that $v_{p}(k-1)>v_{p}(a)$. Then $G\left(p^{e}, k, a\right) \simeq$ $p^{e-s} \mathrm{O}_{p^{s}}$, where

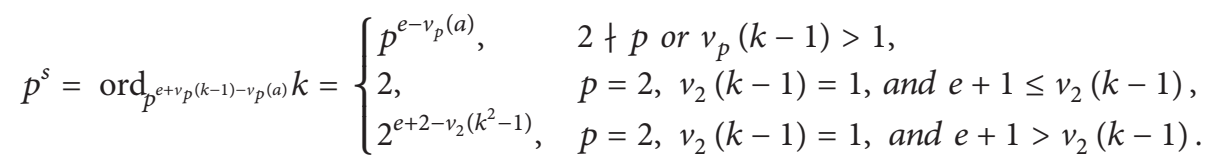

Proof. Let $f(x)=k x+a$. Then $f^{t}(x)=k^{t} x+\left(\sum_{i=0}^{t-1} k^{i}\right) a$. We consider the following congruence equation

$$
k^{t} x+\left(\sum_{i=0}^{t-1} k^{i}\right) a \equiv x \quad(\bmod n) .
$$

Equation (8) has a solution if and only if $\operatorname{gcd}\left(p^{e}, k^{t}-1\right)$ $\operatorname{gcd}\left(p^{e},\left(k^{t}-1\right) /(k-1) \cdot a\right)$. In this case, $p^{e} \mid k^{t}-1$ and $p^{e} \mid$ $\left(k^{t}-1\right) /(k-1) \cdot a$, since $v_{p}(k-1)>v_{p}(a)$. Since $t$ is the minimal positive integer such that $p^{e} \mid\left(k^{t}-1\right) /(k-1) \cdot a$, we get $t=$

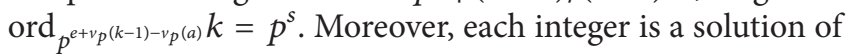


(8). Thus, $G\left(p^{e}, k, a\right)$ is a union of cycles, each having length $p^{s}$. The rest of the proof follows from Lemma 6.

The structure of digraph $G\left(p^{e}, k, a\right)$ is clear now. We classify it as follows.

Type (1): $G\left(p^{e}, k, a\right) \simeq G\left(p^{e}, k\right)$ is a tree attached to a fixed point, which is determined by $\operatorname{gcd}\left(p^{e}, k\right)$.

Type (2): $G\left(p^{e}, k, a\right) \simeq G\left(p^{e}, k\right)$ is a union of cycles, and the minimal length of these cycles is 1 .

Type (3): $G\left(p^{e}, k, a\right) \simeq p^{e-s} O_{p^{s}}$, where $1 \leq s \leq e$.

Let $X_{i}=\left\{p \mid G\left(p^{v_{p}(n)}, k, a\right)\right.$ is type $\left.(i)\right\}$. By the properties of digraph product we see that

$$
G(n, k, a) \simeq G\left(n_{X_{1}}, k, a\right) \times G\left(n_{X_{2}}, k, a\right) \times G\left(n_{X_{3}}, k, a\right),
$$

where $G\left(n_{X_{1}}, k, a\right)$ is a tree attached to a fixed point, $G\left(n_{X_{2}}, k, a\right)$ is a union of cycles, and the minimal length of these cycles is 1 , and $G_{3} \simeq\left(n_{X_{3}} / l\right) O_{l}$.

\section{The Main Results}

We begin with some lemmas before we prove our main results.

Lemma 8. Suppose that $f$ is a permutation of a finite set $X$ and $G(X, f) \simeq \cup_{i=1}^{n} a_{i} O_{i}$. Then $G\left(X, f^{r}\right) \simeq \cup_{i=1}^{n} \operatorname{gcd}(r, i) a_{i} O_{i / \operatorname{gcd}(i, r)}$ for each $r \geq 1$.

Proof. Assume that $E=\left\{x_{1}, x_{2}, \ldots, x_{l}\right\}$ is a cycle of length $l$ in $G(X, f)$ such that $f\left(x_{i}\right)=x_{i+1}$, for $i=1,2, \ldots, l-1$ and $f\left(x_{l}\right)=x_{1}$. Suppose that $x_{i}$ is contained in a cycle of length $m$ in $G\left(X, f^{r}\right)$. Then $m=\min \left\{j: f^{r j}\left(x_{i}\right)=x_{i}\right\}=\min \{j: l \mid$ $r j\}=l / \operatorname{gcd}(r, l)$. Hence, $E$ splits into $\operatorname{gcd}(r, l)$ cycles with the same length $l / \operatorname{gcd}(r, l)$. This completes the proof.

Lemma 9. Let $f_{i}$ be a permutation of a finite set $X_{i}, i=1,2$. Then

(i) $G\left(X_{1}, f_{1}\right) \simeq G\left(X_{2}, f_{2}\right)$ if and only if the numbers of fixed point of $G\left(X_{1}, f_{1}^{r}\right)$ and $G\left(X_{2}, f_{2}^{r}\right)$ are equal for each $r \geq 1$;

(ii) $G\left(X_{1}, f_{1}\right) \times O_{l} \simeq G\left(X_{2}, f_{2}\right) \times O_{l}$ if and only if $G\left(X_{1}, f_{1}^{l}\right) \simeq G\left(X_{2}, f_{2}^{l}\right)$ for each $l \geq 1$.

Proof. Suppose that $G\left(X_{1}, f_{1}\right) \simeq \cup a_{i} O_{i}$ and $G\left(X_{2}, f_{2}\right) \simeq$ $\cup b_{j} \mathrm{O}_{j}$.

Assume that $\varphi: G\left(X_{1}, f_{1}\right) \rightarrow G\left(X_{2}, f_{2}\right)$ is an isomorphism of digraphs. Then $f_{2}(\varphi(x))=\varphi\left(f_{1}(x)\right)$ for any $x \in X_{1}$. By induction, $f_{2}^{r}(\varphi(x))=\varphi\left(f_{1}^{r}(x)\right)$. Hence, $\varphi$ is also an isomorphism of $G\left(X_{1}, f_{1}^{r}\right)$ and $G\left(X_{2}, f_{2}^{r}\right)$. Thus, $A_{1}\left(G\left(X, f_{1}^{r}\right)\right)=$ $A_{1}\left(G\left(X, f_{2}^{r}\right)\right)$. Conversely, if $G\left(X_{1}, f_{1}^{r}\right) \simeq G\left(X_{2}, f_{2}^{r}\right)$, then $a_{1}=b_{1}$. Assume that $a_{i}=b_{i}$ for any $i<r$. By Lemma 8, $A_{1}\left(G\left(X_{1}, f_{1}^{r}\right)\right)=\sum_{i \mid r} i a_{i}$ and $A_{1}\left(G\left(X_{2}, f_{2}^{r}\right)\right)=\sum_{j \mid r} j b_{j}$. Hence, $\sum_{i \mid r} i a_{i}=\sum_{j \mid r} j b_{j}$ and $a_{r}=b_{r}$. The proof of statement (i) is complete by induction.
It remains to prove (ii). Assume that $G(Z, g) \simeq O_{l}$. Then $G\left(X_{i}, f_{i}\right) \times O_{l} \simeq G\left(X_{i} \times Z, f_{i} \times g\right)$, where $f_{i} \times g$ maps $(x, z)$ to $\left(f_{i}(x), g(z)\right)$ for any $(x, z) \in X_{i} \times Z$. Let $G_{i}^{r}=G\left(X_{i} \times Z,\left(f_{i} \times\right.\right.$ $\left.g)^{r}\right)$. By Lemma 8 ,

$$
\begin{aligned}
G_{i}^{r} & \simeq G\left(X_{i}, f_{i}^{r}\right) \times G\left(Z, g^{r}\right) \\
& \simeq G\left(X_{i}, f_{i}^{r}\right) \times \operatorname{gcd}(r, l) O_{l / \operatorname{gcd}(r, l)} .
\end{aligned}
$$

If $G\left(X_{1}, f_{1}\right) \times O_{l} \simeq G\left(X_{2}, f_{2}\right) \times O_{l}$, then $G_{1}^{r} \simeq G_{2}^{r}$ for each $r \geq 1$. Let $r=l$ in (10). We get $G\left(X_{1}, f_{1}^{l}\right) \simeq G\left(X_{2}, f_{2}^{l}\right)$. Conversely, if $G\left(X_{1}, f_{1}^{l}\right) \simeq G\left(X_{2}, f_{2}^{l}\right)$, then by (10) $A_{1}\left(G_{i}^{r}\right)=0$ if $l+r$. If $l \mid r$, then

$$
G_{i}^{r} \simeq G\left(X_{i}, f_{i}^{r}\right) \times \operatorname{gcd}(r, l) O_{l / \operatorname{gcd}(r, l)} \simeq l G\left(X_{i},\left(f_{i}^{l}\right)^{r / l}\right) .
$$

Hence, $G_{1}^{r} \simeq G_{2}^{r}$ if $l \mid r$. Therefore, $A_{1}\left(G_{1}^{r}\right)=A_{1}\left(G_{2}^{r}\right)$ for any $r \geq 1$. By statement (i), $G\left(X_{1}, f_{1}\right) \times O_{l} \simeq G\left(X_{2}, f_{2}\right) \times O_{l}$.

In [3], Sha and $\mathrm{Hu}$ showed that $G\left(p, k_{1}\right) \simeq G\left(p, k_{2}\right)$ if and only if $\operatorname{ord}_{p} k_{1}=\operatorname{ord}_{p} k_{2}$, where $p$ is a prime and $p \nmid k_{i}$. We generalized this result to the following.

Lemma 10. Let $n, k_{1}$ and $k_{2}$ be positive integers such that $\operatorname{gcd}\left(n, k_{i}\right)=1, i=1,2$. Then $G\left(n, k_{1}\right) \simeq G\left(n, k_{2}\right)$ if and only if ord $k_{1}=\operatorname{ord}_{t} k_{2}$ for any divisort of $n$.

Proof. Let $G_{i}^{r}=G\left(n, k_{i}^{r}\right)$. By Lemma 3 and Möbius inverse transform $A_{1}\left(G_{i}^{r}\right)=\operatorname{gcd}\left(k_{i}^{r}-1, n\right)$ for any $r \geq 1$. Suppose that $\operatorname{ord}_{t} k_{1}=\operatorname{ord}_{t} k_{2}$ for any divisor $t$ of $n$. If $t_{1} \mid \operatorname{gcd}\left(k_{1}^{r}-1, n\right)$, let $s=\operatorname{ord}_{t_{1}} k_{1}=\operatorname{ord}_{t_{1}} k_{2}$; then $s \mid r$. Thus $t_{1} \mid \operatorname{gcd}\left(k_{2}^{r}-1, n\right)$. By symmetry, we have $t_{2} \mid \operatorname{gcd}\left(k_{1}^{r}-1, n\right)$ if $t_{2} \mid \operatorname{gcd}\left(k_{2}^{r}-1, n\right)$. Thus, $\operatorname{gcd}\left(k_{1}^{r}-1, n\right)=\operatorname{gcd}\left(k_{2}^{r}-1, n\right)$. By Lemma $9, G\left(n, k_{1}\right) \simeq$ $G\left(n, k_{2}\right)$.

Conversely, if $G\left(n, k_{1}\right) \simeq G\left(n, k_{2}\right)$, then $G_{1}^{r} \simeq G_{2}^{r}$ and $\operatorname{gcd}\left(k_{1}^{r}-1, n\right)=\operatorname{gcd}\left(k_{2}^{r}-1, n\right)$ for any $r \geq 1$. If $t \mid n$, let $s_{i}=\operatorname{ord}_{t} k_{i}$; then $t \mid \operatorname{gcd}\left(k_{1}^{s_{1}}-1, n\right)=\operatorname{gcd}\left(k_{2}^{s_{1}}-1, n\right)$. Hence, $s_{2} \mid s_{1}$. By symmetry, $s_{1} \mid s_{2}$. Thus, $s_{1}=s_{2}$. The proof is complete.

Theorem 11. Let $n=\prod_{i=1}^{r} p_{i}^{e_{i}}$ be the prime factorization of $n$ and let $k_{i}, a_{i}$ be integers, $i=1,2$. Suppose that $Y_{j}=\left\{p_{i} \mid\right.$ $G\left(p_{i}^{e_{i}}, k_{j}, a_{j}\right)$ is Type (2) $\}$ and that the minimal length of cycles in $G\left(n, k_{i}, a_{i}\right)$ is $l_{i}$. Then $G\left(n, k_{1}, a_{1}\right) \simeq G\left(n, k_{2}, a_{2}\right)$ if and only if the following two conditions are satisfied.

(i) $\operatorname{gcd}\left(n, k_{1}\right)=\operatorname{gcd}\left(n, k_{2}\right)$.

(ii) $Y_{1}=Y_{2}=Y, l_{1}=l_{2}=l$, and $\operatorname{ord}_{t} k_{1}^{l}=\operatorname{ord}_{l} k_{2}^{l}$ for any divisort of $n_{Y}$.

Proof. Suppose that $X_{j}=\left\{p_{i} \mid G\left(p_{i}^{e_{i}}, k_{j}, a_{j}\right)\right.$ is Type (1) $\}$ and that $Z_{j}=\left\{p_{i} \mid G\left(p_{i}^{e_{i}}, k_{j}, a_{j}\right)\right.$ is Type (3) $\}$. We have the following decomposition:

$$
\begin{aligned}
G\left(n, k_{i}, a_{i}\right) & \simeq G\left(n_{X_{i}}, k_{i}, a_{i}\right) \times G\left(n_{Y_{i}}, k_{i}, a_{i}\right) \times G\left(n_{Z_{i}}, k_{i}, a_{i}\right) \\
& \simeq G\left(n_{X_{i}}, k_{i}\right) \times G\left(n_{Y_{i}}, k_{i}\right) \times \frac{n_{Z_{i}}}{l_{i}} O_{l_{i}},
\end{aligned}
$$


where $G\left(n_{X_{i}}, k_{i}, a_{i}\right)$ is a tree attached to a fixed point, $G\left(n_{Y_{i}}, k_{i}, a_{i}\right)$ is a union of cycles, and the minimal length of these cycles is 1 .

Therefore, $G\left(n, k_{1}, a_{1}\right) \simeq G\left(n, k_{2}, a_{2}\right)$ if and only if the following two conditions hold.

(iii) $G\left(n_{X_{1}}, k_{1}\right) \simeq G\left(n_{X_{2}}, k_{2}\right)$.

(iv) $n_{Z_{1}} / l_{1}=n_{Z_{2}} / l_{2}$ and $G\left(n_{Y_{1}}, k_{1}\right) \times O_{l_{1}} \simeq G\left(n_{Y_{2}}, k_{2}\right) \times O_{l_{2}}$.

However, by Remark 5, (i) is equivalent to (iii). It remains to show that (ii) is equivalent to (iv). If (ii) is satisfied, then $Z_{1}=Z_{2}$ and by Lemma $10 G\left(n_{Y}, k_{1}^{l}\right) \simeq G\left(n_{Y}, k_{2}^{l}\right)$. By Lemma 9, $G\left(n_{Y}, k_{1}\right) \times O_{l} \simeq G\left(n_{Y}, k_{2}\right) \times O_{l}$. Conversely, if (iv) holds, we have $l_{1}=l_{2}=l$ since the minimal length of cycles in $G\left(n_{Y_{i}}, k_{1}\right) \times O_{l_{i}}$ is $l_{i}$. So $n_{Z_{1}}=n_{Z_{2}}, Z_{1}=Z_{2}, Y_{1}=Y_{2}=Y$, and $G\left(n_{Y}, k_{1}\right) \times O_{l} \simeq G\left(n_{Y}, k_{2}\right) \times O_{l}$. The rest of proof follows from Lemmas 9 and 10.

Remark 12. Suppose that $G\left(n, k_{1}, a_{1}\right) \simeq G\left(n, k_{2}, a_{2}\right)$. By Theorem 11, we see that both $G\left(p^{v_{p}(n)}, k_{1}, a_{1}\right)$ and $G\left(p^{v_{p}(n)}\right.$, $\left.k_{2}, a_{2}\right)$ are the same types for any prime $p \mid n$. Moreover, they are isomorphic if they are Type (1) or (3).

Example 13. Consider $G(40,37,1), G(40,29,1)$, and $G(40$, $29,3)$. One has

$$
\begin{aligned}
G(40,37,1) & \simeq G(5,37,1) \times G(8,37,1) \\
& \simeq G(5,2,1) \times G(8,5,1) \\
& \simeq\left(O_{1} \cup O_{4}\right) \times O_{8} \\
& \simeq 5 O_{8}, \\
G(40,29, i) & \simeq G(5,29, i) \times G(8,29, i) \\
& \simeq G(5,4, i) \times G(8,5, i) \\
& \simeq\left(O_{1} \cup 2 O_{2}\right) \times O_{8} \\
& \simeq 5 O_{8},
\end{aligned}
$$

where $i=1,3$. Hence, $G(40,37,1) \simeq G(40,29,1)$, but $G(5$, $37,1) \neq G(5,29,1)$. This shows that even if $G\left(n, k_{1}\right.$, $\left.a_{1}\right) \simeq G\left(n, k_{2}, a_{2}\right)$, it may happen that $G\left(p^{v_{p}(n)}, k_{1}, a_{1}\right) \neq$ $G\left(p^{v_{p}(n)}, k_{2}, a_{2}\right)$. But we also see that $G(40,29,1) \simeq G(40$, $29,3), G(5,29,1) \simeq G(5,29,3)$, and $G(8,29,1) \simeq G(8,29,3)$. In fact, we have the following result if $k$ is fixed.

Theorem 14. Let $n=\prod_{i=1}^{r} p_{i}^{e_{i}}$ be the prime factorization of $n$. The following three statements are equivalent for any integers $k, a_{1}$, and $a_{2}$ :

(i) $G\left(n, k, a_{1}\right) \simeq G\left(n, k, a_{2}\right)$;

(ii) $\operatorname{gcd}\left(n, k-1, a_{1}\right)=\operatorname{gcd}\left(n, k-1, a_{2}\right)$;

(iii) $G\left(p_{i}^{e_{i}}, k, a_{1}\right) \simeq G\left(p_{i}^{e_{i}}, k, a_{2}\right)$ for each $i \in\{1,2, \ldots, r\}$.

Proof. Let $X_{j}=\left\{p_{i} \mid G\left(p_{i}^{e_{i}}, k, a_{j}\right)\right.$ is Type (1) $\}, Y_{j}=$ $\left\{p_{i} \mid G\left(p_{i}^{e_{i}}, k, a_{j}\right)\right.$ is Type (2) $\}$, and $Z_{j}=\left\{p_{i} \mid G\left(p_{i}^{e_{i}}, k\right.\right.$, $a_{j}$ ) is Type (3)\}. (i) $\Rightarrow$ (ii). By Theorem $11 X_{1}=X_{2}=X, Y_{1}=Y_{2}=Y$, and $Z_{1}=Z_{2}=Z$. If $p_{i} \in X \cup Y$, then $\operatorname{gcd}\left(p_{i}^{e_{i}}, k-1, a_{j}\right)=$ $\operatorname{gcd}\left(p_{i}^{e_{i}}, k-1\right), j=1,2$. If $p_{i} \in Z$, Suppose $G\left(p_{i}^{e_{i}}, k, a_{j}\right) \simeq$ $p_{i}^{e_{i}-s_{i}} O_{p_{i}^{s_{i}}}$ for any $p_{i} \in Z$, where $p_{i}^{s_{i}}=\operatorname{ord}_{p_{i}} \operatorname{evp}_{p_{i}}(k-1)-v_{p_{i}}\left(a_{j}\right) k$. By Lemma 7, $v_{p_{i}}\left(a_{1}\right)=v_{p_{i}}\left(a_{2}\right)$. Thus, $\operatorname{gcd}\left(p_{i}^{e_{i}}, k-1, a_{1}\right)=$ $\operatorname{gcd}\left(p_{i}^{e_{i}}, k-1, a_{2}\right)$ for any $i$. The result follows from that $\operatorname{gcd}\left(n, k-1, a_{j}\right)=\prod_{i=1}^{r} \operatorname{gcd}\left(p_{i}^{e_{i}}, k_{j}, a_{j}\right), j=1,2$.

(ii) $\Rightarrow$ (iii). Assume that $\operatorname{gcd}\left(p_{i}^{e_{i}}, k-1, a_{1}\right)=\operatorname{gcd}\left(p_{i}^{e_{i}}, k-\right.$ $\left.1, a_{2}\right)=p_{i}^{u_{i}}$ for each $i$ with $u_{i} \leq e_{i}$. If $v_{p_{i}}(k-1)=u_{i}$, then by Lemma $4 G\left(p_{i}^{e_{i}}, k, a_{1}\right) \simeq G\left(p_{i}^{e_{i}}, k, a_{2}\right) \simeq G\left(p_{i}^{e_{i}}, k\right)$. If $v_{p_{i}}(k-1)>u_{i}$, then $v_{p_{i}}\left(a_{1}\right)=v_{p_{i}}\left(a_{2}\right)=u_{i}$. By Lemma 7 again $G\left(p_{i}^{e_{i}}, k, a_{j}\right) \simeq p_{i}^{e_{i}-s_{i}} O_{p_{i}^{s_{i}}}$, where $p_{i}^{s_{i}}=\operatorname{ord}_{p_{i}^{e_{i}+v_{p_{i}}(k-1)-u_{i}} k} k$.

(iii) $\Rightarrow$ (i) follows immediately from (2).

\section{The Digraph from Projective Map}

Let $\mathbb{P}^{1}\left(\mathbb{F}_{q}\right)=\mathbb{F}_{q}^{2} \backslash\{(0,0)\} / \sim$ be the one-dimensional projective space over $\mathbb{F}_{q}$, where $\left(a_{1}, a_{2}\right) \sim\left(b_{1}, b_{2}\right)$ if and only if there exists $\lambda \in \mathbb{F}_{q}^{*}$ such that $\lambda a_{i}=b_{i}$. With any invertible $2 \times 2$ matrix $A$ over $\mathbb{F}_{q}$, we associate the Möbius transformation of $\mathbb{P}^{1}\left(\mathbb{F}_{q}\right)$ as $z \mapsto z$ A. Let $f(x)$ be a polynomial over $\mathbb{F}_{q}$ with $f(0) \neq 0$. Recall that $o(f)$, the order of $f(x)$, is the minimal positive integer $d$ such that $f(x) \mid x^{d}-1$. We classify the digraph from Möbius transformation of $\mathbb{P}^{1}\left(\mathbb{F}_{q}\right)$ as follows.

Theorem 15. Let $G=G\left(\mathbb{P}^{1}\left(\mathbb{F}_{q}\right), A\right)$. Suppose that $f(x)$ and $m(x)$ are the characteristic polynomial and minimal polynomial of $A$, respectively. Then

(i) if $f(x)=(x-\lambda)^{2}$ and $m(x)=x-\lambda$, then $G \simeq(q+1) O_{1}$;

(ii) if $f(x)=m(x)=(x-\lambda)^{2}$, then $G \simeq O_{1} \cup(q / p) O_{p}$;

(iii) if $f(x)=\left(x-\lambda_{1}\right)\left(x-\lambda_{2}\right)$ and $\lambda_{1} \neq \lambda_{2}$, then $G \simeq$ $2 O_{1} \cup((q-1) / s) O_{s}$, where $s$ is the multiplicative order of $\lambda_{1} \lambda_{2}^{-1}$;

(iv) if $f(x)$ is irreducible over $\mathbb{F}_{q}[x]$, then $G \simeq((q+1) / s) O_{s}$, where $s=o(f) / \operatorname{gcd}(o(f), q-1)$.

Proof. (i)-(iii). We see that $A$ is similar to $\lambda I_{2},\left(\begin{array}{ll}\lambda & 1 \\ 0 & \lambda\end{array}\right)$ and $\left(\begin{array}{cc}\lambda_{1} & 0 \\ 0 & \lambda_{2}\end{array}\right)$ in cases (i), (ii), and (iii), respectively. But the digraphs are isomorphic from similar matrices. The results follows from a simple calculation.

It remains to prove (iv). Let $\mathbb{K}=\mathbb{F}_{q}[x] /(f(x))$. We can consider $G$ as a subdigraph of $G_{1}=G(\mathbb{K}, A)$ under the natural inclusion $\mathbb{F}_{q} \subseteq \mathbb{K}$. Then $f(x)=(x-\lambda)\left(x-\lambda^{q}\right)$ with some $\lambda$ in $\mathbb{K}$. By (iii), $G_{1} \simeq 2 O_{1} \cup\left(\left(q^{2}-1\right) / t\right) O_{t}$, where $t$ is the multiplicative order of $\lambda^{q-1}$ in $\mathbb{K}$. By a direct computation, we see that $z A \in \mathbb{F}_{q}$ for $z \in \mathbb{K}$ if and only if $z \in \mathbb{F}_{q}$. Hence, $G$ is a union of cycles of $G_{1}$. However, $G$ has no fixed point since $f(x)$ is irreducible. So the length of each cycle in $G$ is $t$, and $G \simeq((q+1) / t) O_{t}$. Note that $t=\min \left\{n>0 \mid \lambda^{n(q-1)}=\right.$ $1\}$, and that $o(f)=\min \left\{n>0 \mid \lambda^{n}=1\right\}$. Therefore, $t=$ $o(f) / \operatorname{gcd}(o(f), q-1)$. This finishes the proof. 
Corollary 16. $G\left(\mathbb{P}^{1}\left(\mathbb{F}_{q}\right), A_{1}\right) \simeq G\left(\mathbb{P}^{1}\left(\mathbb{F}_{q}\right), A_{2}\right)$ if and only if they have the same number of fixed points.

\section{References}

[1] T. D. Rogers, "The graph of the square mapping on the prime fields," Discrete Mathematics, vol. 148, no. 1-3, pp. 317-324, 1996.

[2] C. Lucheta, E. Miller, and C. Reiter, "Digraphs from powers modulo p," The Fibonacci Quarterly, vol. 34, no. 3, pp. 226-239, 1996.

[3] M. Sha and S. Hu, "Monomial dynamical systems of dimension one over finite fields," Acta Arithmetica, vol. 148, no. 4, pp. 309331, 2011.

[4] M. Sha, "Digraphs from endomorphisms of finite cyclic groups," Journal of Combinatorial Mathematics and Combinatorial Computing, vol. 83, pp. 105-120, 2012.

[5] G. Deng and P. Yuan, "Isomorphic digraphs from powers modulo p," Czechoslovak Mathematical Journal, vol. 61, no. 3, pp. 771-779, 2011.

[6] R. A. Hernández Toledo, "Linear finite dynamical systems," Communications in Algebra, vol. 33, no. 9, pp. 2977-2989, 2005. 


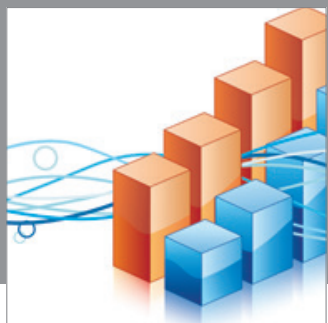

Advances in

Operations Research

mansans

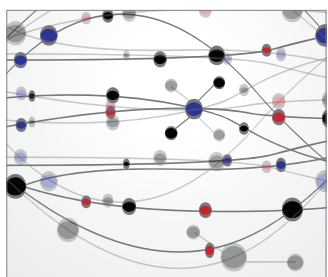

The Scientific World Journal
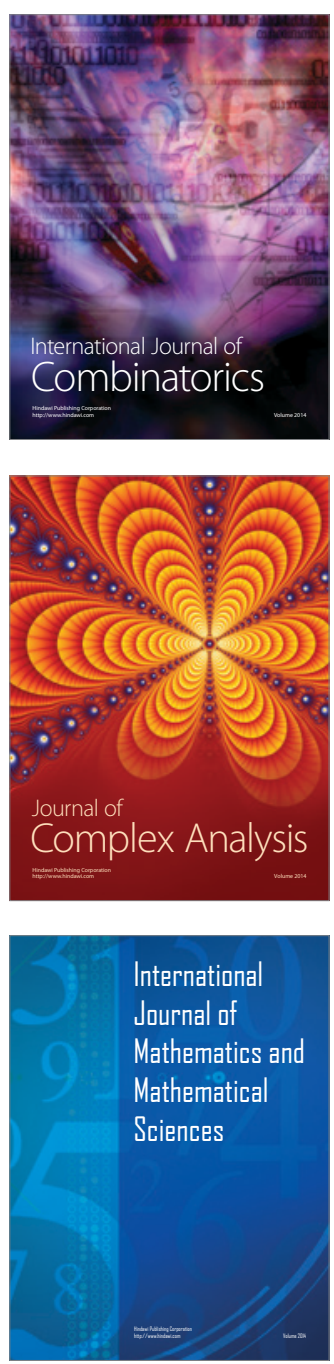
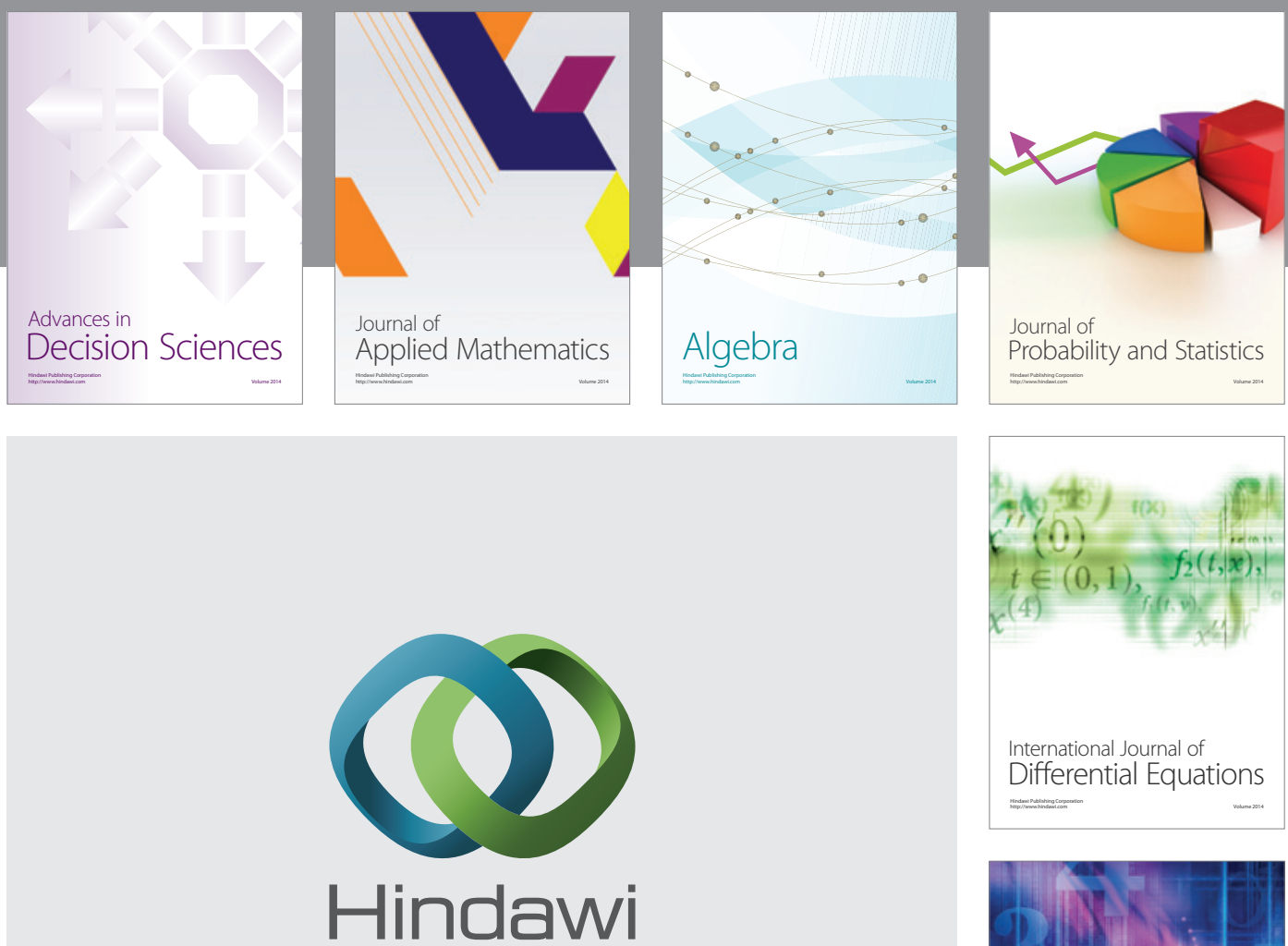

Submit your manuscripts at http://www.hindawi.com
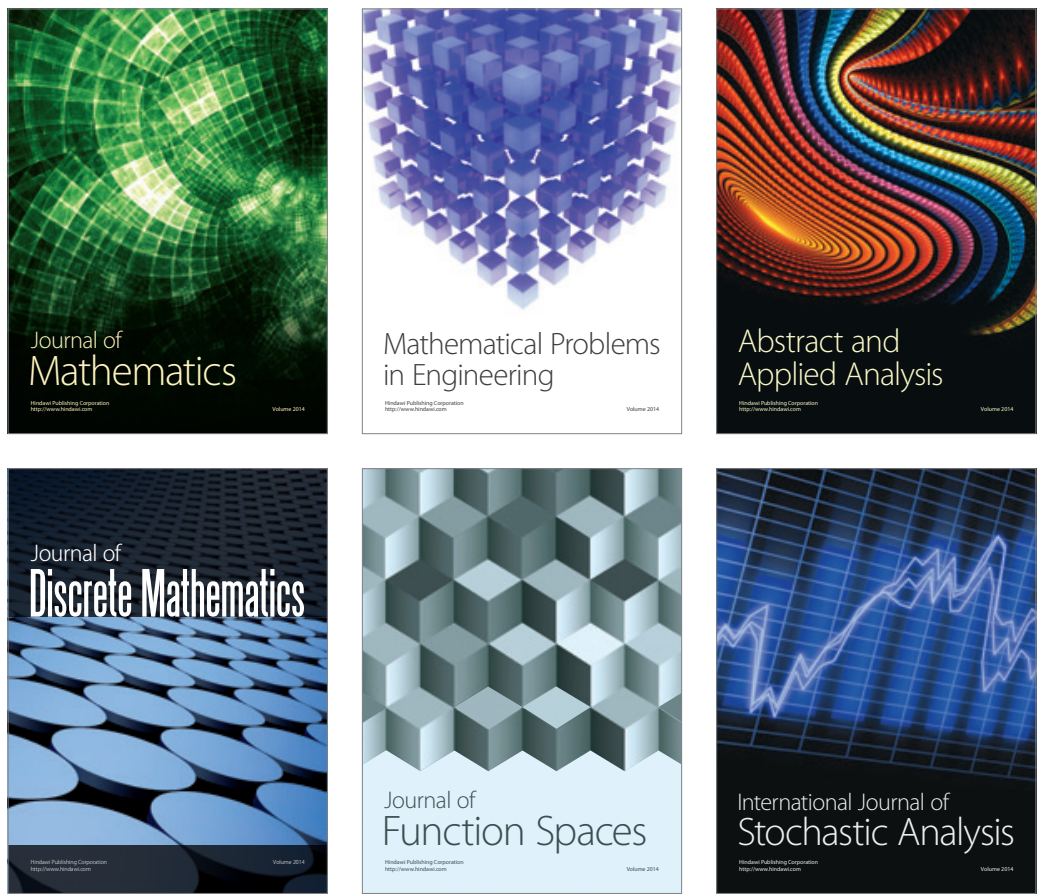

Journal of

Function Spaces

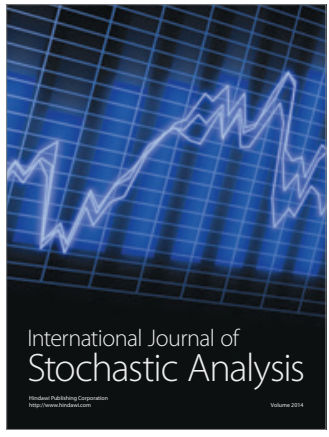

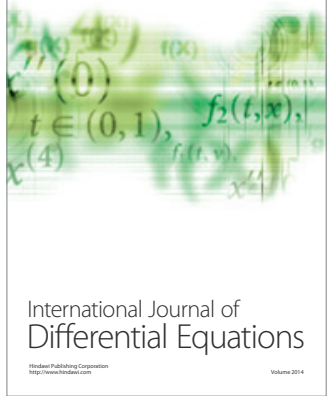
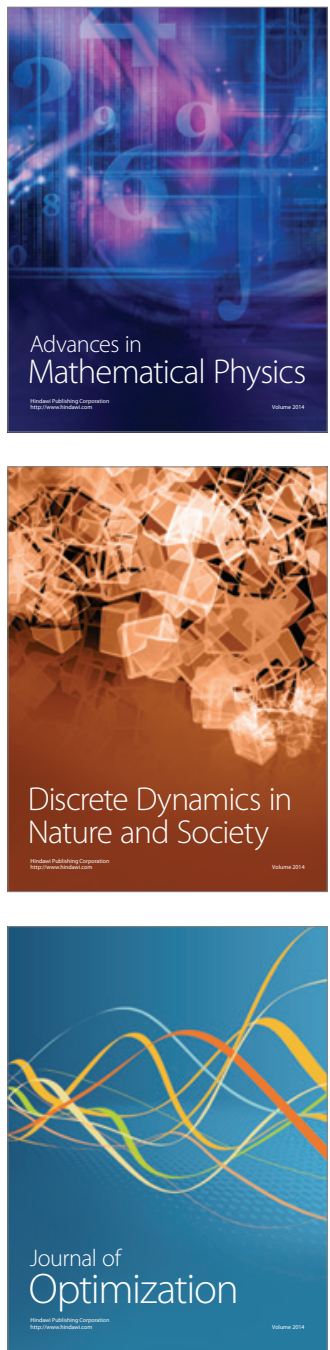